\title{
Post-Bacille Calmette-Guerin surveillance for non-muscle invasive bladder cancer: do random biopsies offer an advantage?
}

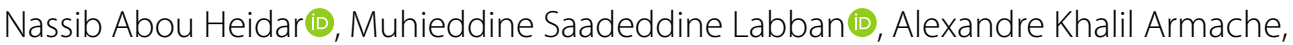

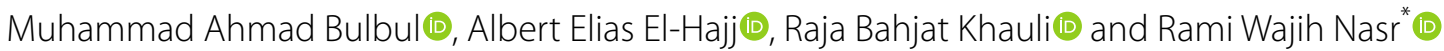

\begin{abstract}
Background: The optimal surveillance method for recurrence of non-muscle invasive bladder cancer (NMIBC) after intravesical BCG treatment is unknown. The aim of this study is to assess the difference between two surveillance methods: cystoscopy with bladder biopsies and office-based flexible cystoscopy in detecting NMIBC recurrence and time to recurrence.

Methods: Charts of patients who underwent transurethral resection of bladder tumor with subsequent intravesical Bacillus Calmette-Guerin (BCG) treatment were reviewed between January 2015 and December 2018. Baseline demographics and oncological parameters were compared between the two methods of surveillance. Then, the role of the surveillance method for NMIBC recurrence and time to recurrence were evaluated in backward logistic regression and hazard ratios estimated in Cox regression models, respectively.

Results: Fifty-one patients (50.5\%) underwent office-based flexible cystoscopy and 50 patients (49.5\%) had bladder biopsies. The patients undergoing either surveillance methods were comparable for baseline demographic and oncological parameter. The predictors of recurrence and earlier BCG relapse were increased body mass index, the presence of multifocal tumors, the presence of concurrent carcinoma in situ, and tumor size at presentation. Bladder cancer recurrence was mostly affected by multifocality of the disease [OR $3.6195 \% \mathrm{Cl}(1.17-11.15)$ ] and the presence of concomitant carcinoma in situ [4.35 (1.29-14.68)]. Yet, the surveillance method neither predicted a higher recurrence yield nor earlier diagnosis.
\end{abstract}

Conclusion: In our cohort, there is neither difference in recurrence yield nor earlier diagnosis of recurrence between office-based flexible cystoscopy and bladder biopsies. Larger prospective studies are needed to assess the generalizability of these findings.

Keywords: Bacillus Calmette-Guerin, Bladder biopsy, Bladder cancer, Non-invasive bladder cancer, Surveillance

\section{Background}

Bladder cancer $(\mathrm{BC})$ is the ninth most commonly diagnosed cancer worldwide with an estimate 81,400 new cases and 17,980 deaths in the USA alone in 2020 [1]. It is

\footnotetext{
*Correspondence: rn05@aub.edu.lb

Department of Surgery, Division of Urology and Renal Transplantation, American University of Beirut - Medical Center, Riad El-Solh, Beirut 1107 2020, Lebanon
}

known to have the highest recurrence rate of any malignancy [1]. The most common bladder malignant tumor is transitional cell carcinoma (TCC), representing more than $90 \%$ of all bladder tumors in the USA [2]. Transitional cell carcinoma is further divided into non-muscle invasive and muscle invasive $\mathrm{BC}$, which has a direct correlation on disease management and prognosis [2].

Non-muscle invasive bladder cancer (NMIBC) is risk-stratified to estimate the likelihood of progression 
to muscle invasive cancer. Several criteria help stratify patients with NMIBC into low-risk, intermediate-risk, and high-risk groups. Patients with high-risk disease, and in some cases intermediate-risk, should undergo intravesical Bacillus Calmette-Guerin (BCG) immunotherapy to decrease recurrence [3].

Patients selected for BCG treatment undergo a 6-week induction course that is followed by clinical surveillance. Disease surveillance is achieved by cystoscopy evaluation, cytology, biomarkers, and biopsy of erythematous lesions. Additionally, bladder biopsies are sometimes performed to evaluate disease progression [4]. New technologies like blue light and fluorescence cystoscopy allow better visualization; however, these modalities are not available in all centers [5].

Although bladder biopsies beget pathological proof of disease or absence of it, this surveillance method is more invasive and costly than the clinic-based flexible cystoscopy evaluation. Besides, there is conflicting data about the ability of bladder biopsies in increasing the yield of recurrence or allowing earlier detection [6]. Therefore, we aim to compare the efficacy of bladder biopsies versus office-based flexible cystoscopy evaluation in detecting NMIBC recurrence.

\section{Methods}

\subsection{Study design}

In order to consider a significant difference in the recurrence rate between the two surveillance modalities, a $20 \%$ difference in proportion of recurrence was chosen. Hence, based on the sample size calculations for a difference in two proportions, a 0.8 power, and an $\alpha$ of 0.05 ; a sample size of at least 97 patients is needed to detect a significant difference in recurrence rates. Therefore, after receiving institutional review board approval, retrospective chart review was done for patients who underwent transurethral resection of bladder tumor (TURBT) at our institution between January 2015 and December 2018. For the designated period, 266 consecutive patients underwent TURBT. Patients with muscle invasive bladder cancer (MIBC) $(n=49)$ and patients with non-malignant lesions $(n=23)$ were excluded. Among patients with $\operatorname{NMIBC}(n=185), 121$ patients fulfilled the criteria for receiving intravesical BCG induction. Then, 20 patients were also excluded for pathologically proven recurrence on the post-induction cystoscopy evaluation at 3 months. Included in the analysis are 50 patients who underwent surveillance by flexible office-based cystoscopy and 51 patients who underwent rigid cystoscopy with bladder cold-cup biopsies (Fig. 1). Surveillance method was performed based on the treating physician's idiosyncratic preference; whereby half of the faculty members $(n=2)$ perform routine bladder biopsies and the other half $(n=2)$ perform office-based cystoscopy. All patients had an ultrasound or computed tomography imaging for evaluation of the bladder lesion. On follow-up, cystoscopic evaluation was performed at 3-month intervals for the first year and later every 6 months. Intravesical BCG (MEDAC) was given to patients with moderate to highrisk features as per the AUA classification [7]. All patients were given an induction course followed by a maintenance course as per the SWOG trial [8]. Moreover, upper tract imaging was performed regularly by computed tomography to monitor for metachronous tumors. Exclusion criteria included patients who were lost to follow-up, those who had no clinical information available, patients who did not receive BCG due to contra-indications, patients with a history of bladder tumors, and patients with low grade tumors or muscle invasive disease, as well as BCG refractory patients (recurrence at 3 months postBCG $n=120$ ). There was no standardized map for the bladder biopsies, and most biopsies were taken from the previous tumor resection site(s). All patients received the standard $81 \mathrm{mg}$ intravesical dose of BCG (MEDAC) once a week for 6 consecutive weeks. All adverse events were classified according to the revised Clavien-Dindo classification [9].

Medical charts were reviewed for clinical, operative, and pathological data including age, gender, medical comorbidities, smoking status, type of surveillance (coldcup biopsies or diagnostic flexible cystoscopy evaluation), tumor stage, grade, patient stratification according to AUA guidelines, tumor multifocality, size of the primary tumor, recurrence, and time to recurrence. A recurrence by flexible cystoscopy was denoted by visualization of papillary growth or suspicious lesions deemed worthy for further investigation followed by pathologic diagnosis. Data on complications due to the intravesical therapy or the TURBT were also recorded [10]. Recurrence was defined as positive results on bladder biopsies or at the time of flexible cystoscopy that were later confirmed with pathology.

\subsection{Statistical analysis}

The patients' demographics and oncological parameters were compared using Mann-Whitney $U$ test for continuous variables and Chi-square or Fisher's exact tests were used for categorical variables. The predictors for NMIBC recurrence were evaluated by a binary logistic regression. Kaplan-Meier survival analysis factored by surveillance method (Log rank test) as well as Cox regression was conducted for the outcome time to recurrence. Both models were performed by the enter method and were adjusted for age, body mass index, hypertension, diabetes, smoking status, tumor size, grade, and stage as well as the surveillance method chosen. The Statistical Package for the 


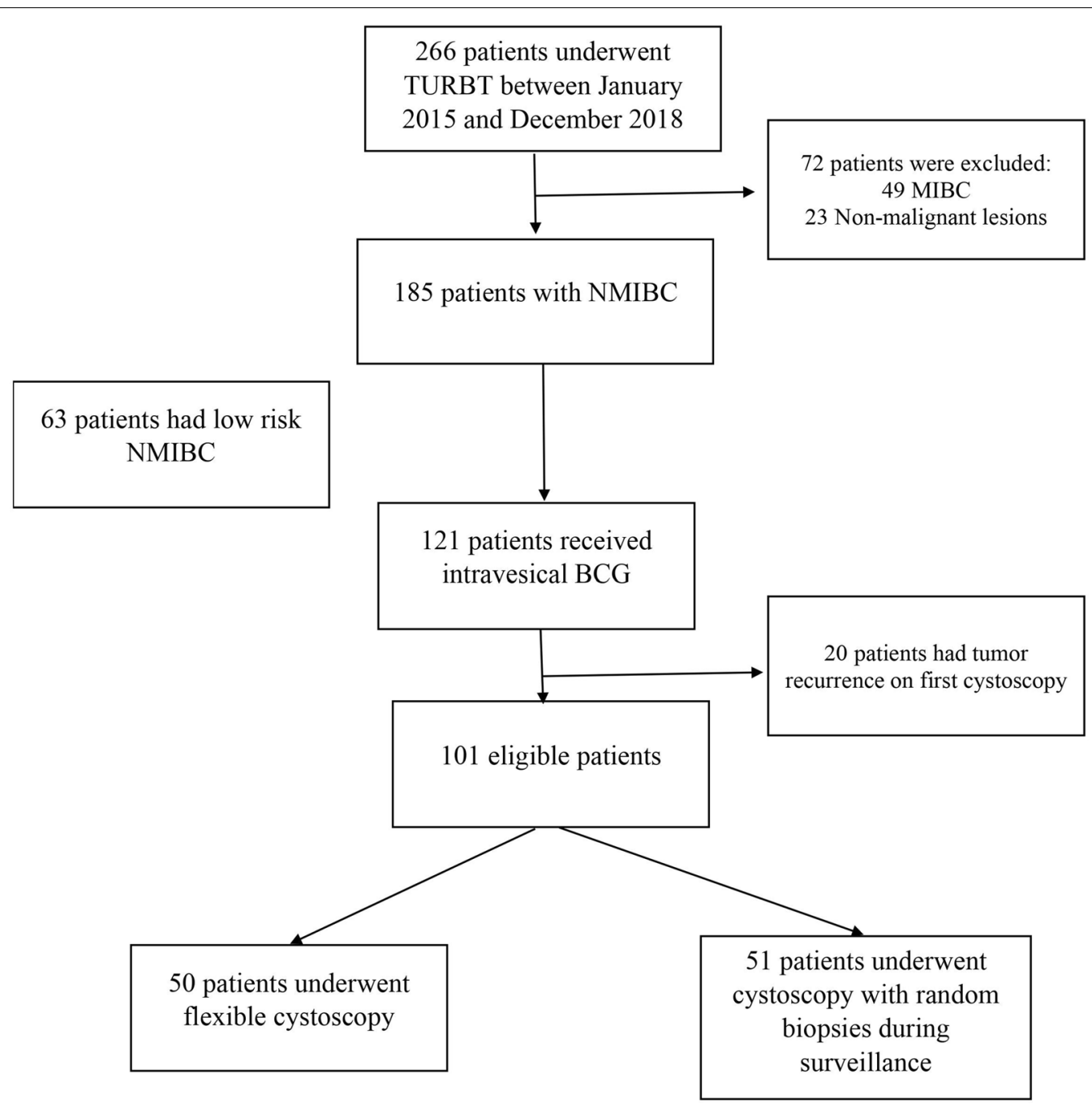

Fig. 1 Flow diagram of study design

Social Sciences (SPSS) version 25 (IBM Corp., Armonk, N.Y., USA) was used to conduct statistical analysis.

\section{Results}

A total of 101 patients underwent surveillance for NMIBC post BCG induction. Fifty-one patients (50.5\%) underwent office-based flexible cystoscopy and 50 patients (49.5\%) had bladder biopsies. Table 1 compares the basic demographics between the two surveillance methods. Both groups were comparable for age, body mass index, hypertension, diabetes, and smoking status $(p>0.05)$. The tumor size was similar for the flexible cystoscopy group $(2.43 \pm 1.1)$ and bladder biopsy group $(2.3 \pm 0.9 ; p=0.453)$. In comparison with bladder biopsy group, the proportion of patients in the flexible cystoscopy group with multifocal tumor, pathological high grade tumor, the presence of concurrent carcinoma in situ (CIS), and stage T1 disease were also similar ( $47.1 \%$ vs. $54.0 \%$; $86.3 \%$ vs. $94.0 \%$; $25.5 \%$ vs. $28.0 \%$; $78.4 \%$ vs. $88.0 \%$, respectively) (Table 1 ). Additionally, the two groups did not differ in AUA risk distribution $(p=0.1)$.

Since the median was not reached in either group, the 75th percentile survival was $18 \pm 1$ months for the flexible cystoscopy group and $14 \pm 3$ months for the bladder biopsy group (Fig. 2). The surveillance method did not impact the yield of recurrence (Mantel-Cox $p=0.5$ ). At the multivariate level, every one-unit increase in BMI resulted in $23 \%$ increase odds and 13\% increase hazard of NMIBC recurrence (logistic regression) and earlier recurrence (Cox Regression), respectively. In the logistic regression, recurrence was also predicted by the presence of concurrent CIS [OR 4.35 95\% CI (1.29-14.68)], 
Table 1 Baseline demographics and oncological parameters in patients who underwent either flexible cystoscopy or cystoscopy with repeat biopsies for non-muscle invasive bladder cancer

\begin{tabular}{llll}
\hline Variables & $\begin{array}{l}\text { Flexible } \\
\text { cystoscopy } \\
(\mathrm{N}=51)\end{array}$ & Repeat Biopsy (N=50) & $p$ value \\
& $\mathrm{N}(\%) ; \bar{x} \pm$ SD & $\mathrm{N}(\%) ; \bar{x} \pm$ SD & \\
\hline Demographics & & & \\
Age (years) & $66.2 \pm 12.2$ & $65.5 \pm 10.0$ & 0.5 \\
Male & $43(84.3 \%)$ & $42(84.0 \%)$ & 0.9 \\
BMI (kg/m $\left.{ }^{2}\right)$ & $27.4 \pm 3.4$ & $26.9 \pm 4.4$ & 0.5 \\
Hypertension & $36(70.6 \%)$ & $36(72.0 \%)$ & 0.9 \\
Diabetes & $11(21.6 \%)$ & $14(28.0 \%)$ & 0.5 \\
Smoking & $30(58.8 \%)$ & $25(50.0 \%)$ & 0.4 \\
Oncological variables & & 0.5 \\
Tumor size (cm) & $2.43 \pm 1.1$ & $2.3 \pm 0.9$ & 0.5 \\
Multifocal & $24(47.1 \%)$ & $27(54.0 \%)$ & 0.3 \\
High grade & $44(86.3 \%)$ & $47(94.0 \%)$ & 0.8 \\
Concurrent CIS & $13(25.5 \%)$ & $14(28.0 \%)$ & 0.2 \\
Stage T1 & $40(78.4 \%)$ & $44(88.0 \%)$ & 0.147 \\
AUA risk stratification & & & \\
Moderate risk & $13(25.5 \%)$ & $7(14.0 \%)$ & $43(86.0 \%)$ \\
High risk & $38(74.5 \%)$ & & \\
\hline
\end{tabular}

multifocal tumors at presentation [3.61; (1.17-11.15)], and tumor size [2.23 (1.23-4.03)] (Table 2). Furthermore, in the Cox regression, earlier recurrence was more likely in patients with concurrent CIS [2.46 (1.08-5.58)]. Besides, every one-centimeter increase in size of tumor results in $52 \%$ increased hazard of earlier recurrence. Nevertheless, the surveillance method neither predicted recurrence (logistic regression) nor the earlier diagnosis of recurrence (Cox regression).

\subsection{Complications}

No serious complications were encountered (no Clavien III or IV complications were recorded). Of the patients undergoing TURBT, 5 patients had prolonged bleeding (3\%), $2(1 \%)$ patients had post-operative urinary infection, $6(4 \%)$ patients had urinary retention. Only $5(5 \%)$ patients developed a low-grade fever secondary to BCG treatment. Yet, no patient discontinued the course due to side effects. Patients who underwent flexible cystoscopy or cystoscopy with bladder biopsies had no procedure specific grade II or higher complications. No mortalities were encountered in the follow-up of included patients.

\section{Discussion}

Guidelines for the management of NMIBC address the disease's biological inclination for recurrence or progression $[4,11]$. Therefore, we sought to determine the optimal NMIBC surveillance method post BCG induction: whether to perform bladder biopsies in the operating room or to resort to office-based flexible cystoscopy. While bladder biopsy surveillance has an added cost on the healthcare system, results in discomfort to the patient, and augments procedural risks; surveillance flexible cystoscopy begets no objective patho-oncological feedback.

There are multiple risk factors, whether tumor-specific or patient-specific, that can predict NMIBC recurrence. In our cohort, patients who experienced a recurrence had a higher BMI. A higher BMI is associated with hormonal changes and systemic inflammatory response increasing the risk of recurrence and progression of NMIBC [12]. Although the current smoking status was shown to be associated with recurrence, this was not the case in our study [13].

Initial tumor size, tumor multifocality, tumor grade, history of recurrence, a shorter timing of intravesical therapy, and presence of CIS are all disease specific variables associated with recurrence [14-16]. Similarly, in our cohort, tumor size, tumor multifocality, and the presence of concomitant CIS were associated with higher odds of recurrence and time to recurrence for NMIBC recurrence. Tumor size and tumor mutlifocality make it harder for complete surgical resection [14]. Furthermore, multifocality implies a biological predisposition where the entire transitional epithelium is subject to genetic instability [14].

Although bladder biopsies have been found to increase the yield and tumor upstaging at the time of initial TURBT, the literature is divided regarding its role in the surveillance stage $[17,18]$. While May et al. found that bladder biopsies had a positive impact on therapeutic decisions of all NMIBC patients, Highshaw et al. found that bladder biopsies are unnecessary in case of negative findings on cystoscopy $[17,19]$. Similarly, biopsies were found to be unwarranted when cytology and flexible cystoscopy are combined for surveillance [20,21]. To the authors' knowledge, this study is the first to assess the time to recurrence when comparing bladder biopsies to flexible cystoscopy. In our cohort, bladder biopsies were as effective as office-based cystoscopy as a surveillance method in terms of recurrence detection or even earlier NMIBC uncovering. Despite the fact that both bladder biopsies and flexible cystoscopy were equally safe and not associated with any grade II or higher Clavien complications, surveillance with bladder biopsies subjects patients to unnecessary anesthesia and added cost [22].

New technologies such as narrow band imaging and blue light cystoscopy increase the yield of detection $[23,24]$. However, most developing countries lack these new technologies due to cost issues as well as due to the 


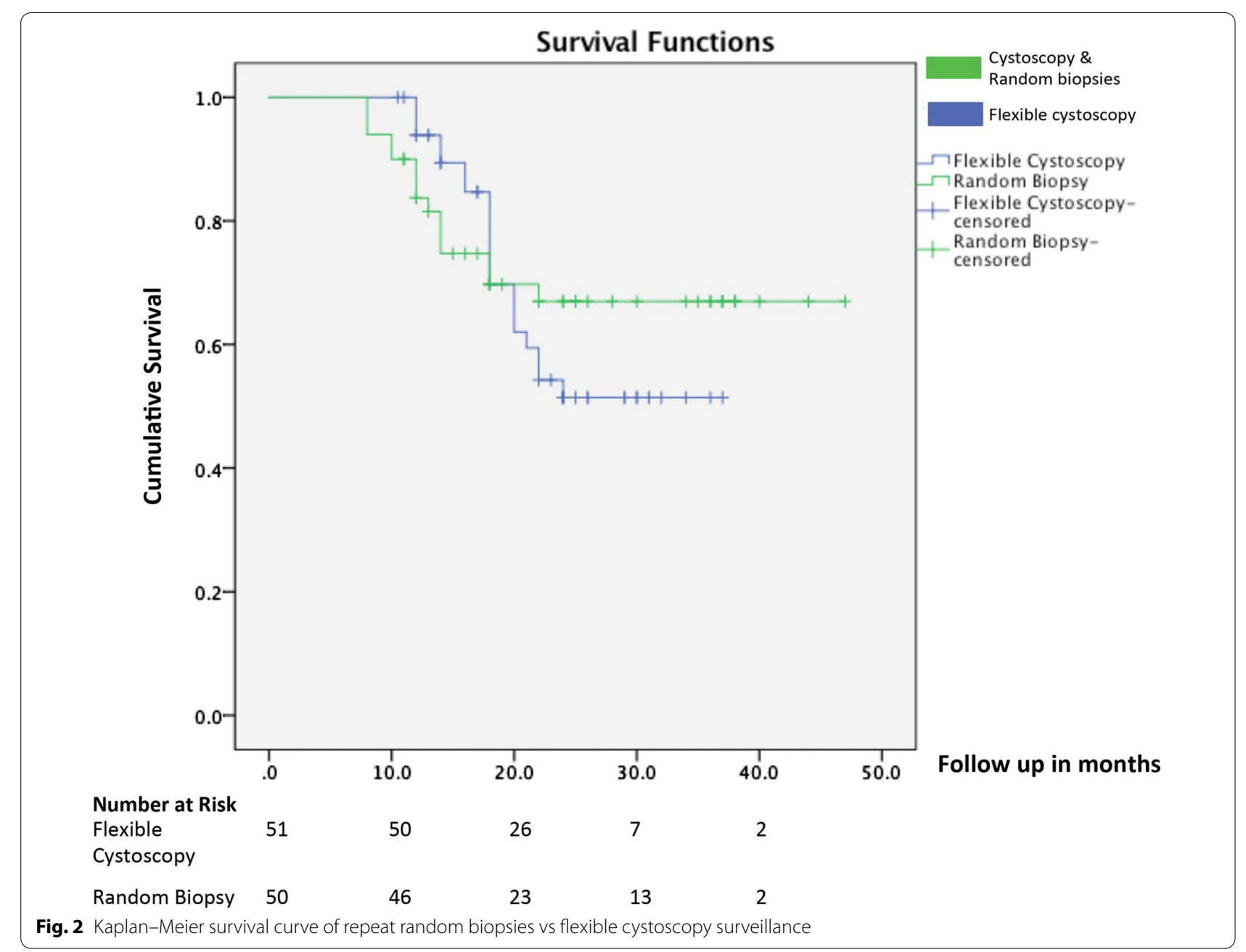

Table 2 Predictors of recurrence and time to recurrence in patients with non-invasive muscle bladder cancer

\begin{tabular}{|c|c|c|}
\hline Variable & $\begin{array}{l}\text { Recurrence }^{a} \\
\text { Odds ratio }(95 \% \text { Cl) }\end{array}$ & $\begin{array}{l}\text { Time to recurrence } \\
\text { Hazard ratio }(95 \% \mathrm{Cl})\end{array}$ \\
\hline Age & $0.99(0.94-1.04)$ & $1.00(0.96-1.04)$ \\
\hline Body mass index $\left(\mathrm{kg} / \mathrm{m}^{2}\right)$ & $1.23(1.06-1.43)$ & $1.13(1.03-1.23)$ \\
\hline Hypertension & $1.04(0.30-3.56)$ & $1.29(0.53-3.12)$ \\
\hline Diabetes & $0.96(0.29-3.23)$ & $0.83(0.32-2.16)$ \\
\hline Smoking & $1.15(0.40-3.37)$ & $0.94(0.44-2.02)$ \\
\hline Surveillance method ${ }^{c}$ & $0.63(0.22-1.80)$ & $0.97(0.48-2.00)$ \\
\hline Size $(\mathrm{cm})$ & $2.23(1.23-4.0)$ & $1.52(1.08-2.14)$ \\
\hline Concurrent CIS & $4.35(1.29-14.68)$ & $2.46(1.08-5.58)$ \\
\hline Multifocality & $3.61(1.17-11.15)$ & $1.93(0.83-4.47)$ \\
\hline High grade tumor ${ }^{d}$ & $2.17(0.39-12.20)$ & $1.06(0.34-3.36)$ \\
\hline Stage $T 1^{\mathrm{e}}$ & $0.40(0.08-1.96)$ & $0.57(0.20-1.59)$ \\
\hline
\end{tabular}

a Binary logistic regression (Enter Method)

${ }^{\mathrm{b}}$ Estimated coefficients in the Cox proportional hazards regression model (Enter Method)

c Flexible cystoscopy as reference to cystoscopy with repeat biopsy

d Low grade tumor as a reference to high grade tumor

e $\mathrm{Ta}$ as a reference to $\mathrm{T} 1 \mathrm{~s}$ marginal improvement these tools bring about in overall outcome from a public health standpoint. Moreover, urinary biomarkers have been on the rise recently in the surveillance of NMIBC, but these are currently under investigation and are not for use in clinical practice [24].

\subsection{Limitations}

Although the data was retrospectively collected, the cohort encompassed non-selected consecutive patients presenting at our tertiary care center. Despite the relatively small sample size, our site is a referral center of neighboring countries ensuring the diversity of our population. The surveillance was performed by different urologists at our institution with each their preference of the method. Additionally, there was no standardized mapping of the bladder biopsies performed and the number of biopsies taken was not recorded in both methods. Furthermore, cystoscopy findings were extracted from operative reports and not from videotaped procedures. Also, 
in cases of flexible cystoscopy surveillance ascertainment of recurrence has to be with a pathologic specimen.

\section{Conclusion}

NMIBC is a disease characterized by a high recurrence rate. In our cohort, bladder biopsies for surveillance of patients with history of NMIBC neither offered an increased detection of recurrence nor an earlier uncovering. Yet, larger cohorts and prospective studies are needed to assess the generalizability of our findings.

\section{Abbreviations}

BCG: Bacillus Calmette-Guerin; BC: Bladder cancer; MIBC: Muscle invasive bladder cancer; NMIBC: Non-muscle invasive bladder cancer; TCC: Transitional cell carcinoma; TURBT: Transurethral resection of bladder tumor.

\section{Acknowledgements}

None.

\section{Authors' contributions}

$\mathrm{NAH}$ and RN contributed to concept; $\mathrm{NAH}$ contributed to design; AE and RK contributed to supervision; ML contributed to data collection and/or processing and analysis and/or interpretation; $\mathrm{ML}$ and $\mathrm{AA}$ were involved in literature search; NA, MB, and RK contributed to writing manuscript; $M B, A E, R K$, and $\mathrm{RN}$ contributed to critical review. All authors have read and approved the manuscript.

\section{Funding}

None.

\section{Availability of data and materials}

Available upon request.

\section{Declarations}

\section{Ethics approval and consent to participate}

This is a study that received Institutional Review Board approval at the American University of Beirut (AUB-IRB-BIO-0069). Consent does not apply since this is a retrospective chart review.

\section{Consent for publication}

This study is a retrospective chart review and does not require individual consent from the participants.

\section{Competing interests}

The authors have no conflicts of interest to declare.

Received: 26 January 2021 Accepted: 5 March 2021

Published online: 17 March 2021

\section{References}

1. Siegel RL, Miller KD, Jemal A (2020) Cancer statistics, 2020. CA Cancer J Clin 70(1):7-30

2. Babjuk M, Bohle A, Burger M et al (2017) EAU guidelines on non-muscleinvasive urothelial carcinoma of the bladder: update 2016. Eur Urol 71(3):447-461

3. Josephson DY, Pasin E, Stein JP (2006) Superficial bladder cancer: part 1. Update on etiology, classification and natural history. Expert Rev Anticancer Therapy 6(12):1723-1734

4. Woldu SL, Bagrodia A, Lotan Y (2017) Guideline of guidelines: non-muscleinvasive bladder cancer. BJU Int 119(3):371-380

5. Karaoglu I, van der Heijden AG, Witjes JA (2014) The role of urine markers, white light cystoscopy and fluorescence cystoscopy in recurrence, progression and follow-up of non-muscle invasive bladder cancer. World J Urol 32(3):651-659

6. van der Meijden A, Oosterlinck W, Brausi M et al (1999) Significance of bladder biopsies in Ta, T1 bladder tumors: a report from the EORTC GenitoUrinary Tract Cancer Cooperative Group. Eur Urol 35(4):267-271

7. Association AU (2016) Diagnosis and Treatment of Non-Muscle Invasive Bladder Cancer: AUASUO Joint Guideline [Guidelines]. auanet.org2016 [cited AUASSO Joint Guideline: Published 2016 April 25th 2020]. https:// www.auanet.org/guidelines/bladder-cancer-non-muscle-invasive-guide line-nmibcrisktable

8. Kamat AM, Flaig TW, Grossman HB et al (2015) Consensus statement on best practice management regarding the use of intravesical immunotherapy with BCG for bladder cancer. Nat Rev Urol 12(4):225-235

9. Dindo D, Demartines N, Clavien P-A (2004) Classification of surgical complications: a new proposal with evaluation in a cohort of 6336 patients and results of a survey. Ann Surg 240(2):205

10. Sylvester RJ, van der Meijden AP, Oosterlinck W et al (2006) Predicting recurrence and progression in individual patients with stage Ta T1 bladder cancer using EORTC risk tables: a combined analysis of 2596 patients from seven EORTC trials. Eur Urol 49(3):466-477

11. Babjuk M, Burger M, Zigeuner R et al (2013) EAU guidelines on nonmuscle-invasive urothelial carcinoma of the bladder: update 2013. Eur Urol 64(4):639-653

12. Westhoff E, Witjes JA, Fleshner NE et al (2018) Body mass index, diet-related factors, and bladder cancer prognosis: a systematic review and meta-analysis. Bladder Cancer (Amsterdam, Netherlands) 4(1):91-112

13. Li HM, Azhati B, Rexiati M et al (2017) Impact of smoking status and cumulative smoking exposure on tumor recurrence of non-muscle-invasive bladder cancer. Int Urol Nephrol 49(1):69-76

14. Liu S, Hou J, Zhang H et al (2015) The evaluation of the risk factors for non-muscle invasive bladder cancer (NMIBC) recurrence after transurethral resection (TURBt) in Chinese population. PLoS ONE 10(4):e0123617

15. Shen Z, Xie L, Chen T et al (2016) Risk factors predictive of recurrence and progression for patients who suffered initial recurrence after transurethral resection of stage $\mathrm{P} T 1$ bladder tumor in Chinese population: a retrospective study. Medicine 95(5):e2625

16. Gontero P, Sylvester R, Pisano F et al (2015) Prognostic factors and risk groups in T1G3 non-muscle-invasive bladder cancer patients initially treated with Bacillus Calmette-Guerin: results of a retrospective multicenter study of 2451 patients. Eur Urol 67(1):74-82

17. May F, Treiber U, Hartung R et al (2003) Significance of random bladder biopsies in superficial bladder cancer. Eur Urol 44(1):47-50

18. Thorstenson A, Schumacher MC, Wiklund NP et al (2010) Diagnostic random bladder biopsies: reflections from a population-based cohort of 538 patients. Scand J Urol Nephrol 44(1):11-19

19. Highshaw RA, Tanaka ST, Evans CP et al (eds) (2003) Is bladder biopsy necessary at three or six months post BCG therapy? Urologic Oncology Seminars and Original Investigations. Elsevier, Amsterdam

20. Guy L, Savareux L, Molinié V et al (2006) Should bladder biopsies be performed routinely after bacillus Calmette-Guérin treatment for high-risk superficial transitional cell cancer of the bladder? Eur Urol 50(3):516-520

21. Swietek N, Waldert M, Rom M et al (2012) The value of transurethral bladder biopsy after intravesical bacillus Calmette-Guérin instillation therapy for nonmuscle invasive bladder cancer: a retrospective, single center study and cumulative analysis of the literature. J Urol 188(3):748-753

22. Soria F, Droller MJ, Lotan Y et al (2018) An up-to-date catalog of available urinary biomarkers for the surveillance of non-muscle invasive bladder cancer. World J Urol 36(12):1981-1995

23. Daneshmand S, Patel S, Lotan Y et al (2018) Efficacy and safety of blue light flexible cystoscopy with hexaminolevulinate in the surveillance of bladder cancer: a phase III, comparative, multicenter study. J Urol 199(5):1158-1165

24. Xiong Y, Li J, Ma S et al (2017) A meta-analysis of narrow band imaging for the diagnosis and therapeutic outcome of non-muscle invasive bladder cancer. PLoS ONE 12(2):e0170819

\section{Publisher's note}

Springer Nature remains neutral with regard to jurisdictional claims in published maps and institutional affiliations. 